\title{
Plataformas virtuales: ¿Herramientas para el aprendizaje? \\ Las diferencias entre ADAN y EVA
}

\author{
Mauricio Nihil Olivera Cajiga ${ }^{1}$ \\ María Julia Morales² \\ Analía Passarini ${ }^{3}$ \\ Natalia Correa ${ }^{4}$
}

Resumen: Este artículo analiza las apropiaciones ${ }^{5}$ de Internet que establecen los universitarios de la Facultad de Comunicación de la Universidad de la República de Uruguay (UdelaR), así como sus prácticas y usos en las plataformas virtuales educativas. Se observa: a) la participación; b) la colaboración; y c) la gestión compartida de recursos que hacen los estudiantes de las plataformas ADAN (creada por ellos) y EVA (creada por Udelar). El estudio es exploratorio ${ }^{6}$ e incorpora técnicas cuantitativas y cualitativas que cubren una encuesta a los estudiantes, entrevistas en profundidad a los profesores, la observación de ambas plataformas y análisis de datos secundarios. El artículo concluye con la discusión que sitúa las prácticas y las lógicas de la red como un espacio que resignifica e interpela a sus actores y a las lógicas centralizadas y jerarquizadas universitarias. Palabras clave: plataformas virtuales; Internet; estudiantes; participación; trabajo colaborativo

\footnotetext{
1 Universidad de la República. Montevidéu, Uruguai. E-mail: mauricio.nihil@fic.edu.uy

2 Universidad de la República. Montevidéu, Uruguai. E-mail: mjmorgonz@gmail.com

3 Universidad de la República. Montevidéu, Uruguai. E-mail: analia.passarini@fic.edu.uy

4 Universidad de la República. Montevidéu, Uruguai. E-mail: natalia.correa@fic.edu.uy

5 Nos referimos al conjunto de procesos socioculturales que intervienen en el uso, la socialización y la significación de las nuevas tecnologías en diversos grupos sociales.

6 Martín Motta, docente en Comunicación Social de la Facultad de Información y Comunicación (Udelar) actuó como investigador colaborador en el estudio exploratório.
} 
Resumo: Este artigo analisa as apropriações7 que os universitários da Faculdade de Comunicação da Universidade da República do Uruguai estabelecem com a internet, assim como suas práticas e usos nas plataformas virtuais educativas. Observamos: a) a participação; b) a colaboração; c) a gestão compartilhada de recursos que os estudantes fazem nas plataformas ADAN (criada por eles) $e$ EVA (criada por Udelar). O estudo é exploratório e incorpora técnicas quantitativas e qualitativas que abrange entrevista com os estudantes, entrevistas em profundidade com os professores, obsevação de ambas plataformas e análise de dados secundários. O artigo conclui com uma reflexão que situa as práticas e as lógicas da rede como um espaço que ressignifica e interpela seus atores e as lógicas universitárias centralizadas e hierquizadas.

Palavras-chave: plataformas virtuais; internet; estudantes; participação; trabatho colaborativo

7 Nos referimos ao conjunto de processos socioculturais que intervêm no uso, na socialização e na significação das novas tecnologias em diversos grupos sociais. 


\section{Introducción}

Partimos en este artículo del supuesto que estamos ante un nuevo escenario, uno hipertextual, reticular, lleno de nodos. En ese escenario, el sistema educativo formal comenzó a sentirse interpelado, en gran parte por un cambio de paradigma en cuanto a las formas de apropiación del conocimiento. El rol del docente y estudiante, los tipos de conocimientos impartidos y las formas de aprender están en constante cuestionamiento. Un importante protagonista de los cambios en ese contexto es Internet, un sistema digital que se caracteriza por una arquitectura descentralizada, por una multiplicación de fuentes de emisión, disponibilidad ininterrumpida de datos, sonidos, imágenes y utilización simultánea en interacciones singulares. Denis de Moraes (2007, p. 2) entiende que Internet habilita redes que "se distinguen como sistemas organizacionales con estructuras flexibles y colaborativas basadas en afinidades, objetivos y temáticas comunes entre los miembros".

Las redes en Internet, como explica de Moraes, descentró la relación docente-estudiante dentro del sistema educativo que aún se sostiene en el esquema clásico de enseñanza universitaria y en el cual el saber conserva un doble carácter: centralizado y personificado. En palabras de Martín Barbero (2007, p. 12) "la escuela está dejando de ser el único lugar de legitimación del saber ya que hay una multiplicidad de saberes que circulan por otros canales, difusos y descentralizados".

Autores como Darling-Hammond (2001) sostienen que el rol del docente en la sociedad actual adquirió una mayor complejidad, que ya no alcanza con dominar la especialidad que se imparte. El compromiso del profesor, con las TIC trasciende la transmisión de un saber concreto, lo que le supone estudio, valor y esfuerzo para poner en juego todas las capacidades de crear, cooperar e innovar en sus prácticas pedagógicas.

La revolución tecnológica introdujo en nuestras sociedades, como apunta Castells (1997), más que una cantidad inusitada de nuevas máquinas, un nuevo modo de relación entre los procesos simbólicos (que constituyen lo cultural) y las formas de producción y distribución de los bienes y servicios, un nuevo modo de producir asociado a un nuevo 
modo de comunicar. El lugar de la cultura en la sociedad cambia cuando la mediación tecnológica (ECHEVERRÍA, 1999) de la comunicación deja de ser solo instrumental para densificarse y volverse estructural. La tecnología no debe ser vista como nuevos aparatos sino como nuevos modos de percepción y de lenguaje, sensibilidades y escrituras, nuevas formas de relacionamiento y aprendizaje. Si acordamos que existe una transformación en los modos de circulación del saber, tal como sugieren diversos autores (RIFKIN, 2000; CASTELLS, 1997; FISCHER, 2001), podemos plantear entonces que comenzamos a atravesar una profunda transformación social.

Este artículo pretende avanzar en el conocimiento sobre los sentidos y significados que adquieren para los estudiantes y los docentes el uso de las plataformas virtuales de aprendizaje en el ámbito de las asignaturas de la Facultad de la Información y Comunicación de la UdelaR. Para ello partimos de las siguientes preguntas de investigación: ¿Las plataformas de aprendizaje en Internet son simplemente un nuevo instrumento que agiliza, refuerza y permite hacer mejor las tareas que ya se realizaban? ¿O el conjunto de los cambios expuestos que encarnan esas plataformas en Internet estarían provocando la ruptura con muchos de los sistemas clásicos de enseñanza en los que se basa la educación y la intermediación administrativa en el campo de la gestión del conocimiento? ¿Cuáles son las dimensiones, la profundidad y cambio que generan estas plataformas en la intermediación educacional? ¿La lógica de la red interpela las lógicas tradicionales, centralizadas y jerarquizadas de la institución educativa y de sus actores?

Para explorar las relaciones que habilita Internet dentro de la educación superior y en la gestión del conocimiento se observan dos plataformas que funcionan de forma virtual: EVA (Moodle), gestionada por la UdelaR desde 2008, y ADAN (Wordpress) desarrollada y gestionada por los propios estudiantes del Instituto de Comunicación de la Facultad de Información y Comunicación (FIC) desde 2015.

La Universidad de la República (UdelaR) es la principal universidad pública uruguaya y la que concentra la mayor parte del estudiantado 
universitario: de una matrícula total de 107.666 estudiantes universitarios para el 2014, 85.905 pertenecen a la UdelaR (MEC, 2014). La enseñanza se ha desarrollado históricamente de manera presencial y con una marcada tendencia a las estrategias expositivas, tradición que aún sobrevive en el aula. Si bien desde finales del siglo pasado hubo intentos de generar políticas que tiendan a la incorporación de tecnologías en las aulas y a la diversificación de modalidades de cursado alternativas (semi-presencialidad, educación a distancia) fueron las políticas desarrolladas a partir de la ejecución del Proyecto "Generalización del uso educativo de las tecnologías de la información y la comunicación en la Universidad de la República" (TICUR), ejecutado entre 2008 y 2010, las que lograron colocar el tema en la agenda. Con este proyecto se logró, por ejemplo, el desarrollo de una red de Entornos Virtuales de Aprendizaje (EVA) que utilizan Moodle como plataforma educativa. La plataforma EVA, a la cual tienen acceso todos los estudiantes universitarios de la UdelaR, fue implementada con el fin de integrar a las TIC en la enseñanza universitaria:

La plataforma ofrece varias funcionalidades que sirven de apoyo a las estrategias de enseñanza, investigación y extensión, entre las que se destacan: creación y gestión de cursos, creación de espacios para grupos de trabajo, comunicación, creación colectiva, gestión de usuarios, aprendizaje colaborativo y herramientas de evaluación" (Universidad de la República (Uruguay). Comisión Sectorial de Enseñanza, 2012) ${ }^{8}$

El sitio permite: a) acceder a los materiales de estudio en sus diferentes formatos (texto, audio, multimedia, etc.) y b) la interacción entre estudiantes y docentes a través de correo electrónico, chat, foros, etc. Además, desde el mes de julio de 2014, incorporó una Sala Multimedia del Programa de Entornos Virtuales de Aprendizaje (ProEVA) que cuenta con un estudio de grabación de video, un repositorio multimedia y un sistema de grabación de clases. La idea de estas herramientas es incentivar la generación de recursos virtuales por parte de los docentes

8 Las negritas son nuestras. 
y otorgar un marco de formación y de accesibilidad de infraestructura adecuados.

La plataforma ADAN fue desarrollada por los propios estudiantes en 2015 (su nombre es un juego irónico en relación a la plataforma universitaria EVA), cuyo propósito es fomentar el vínculo y la articulación de los estudiantes a partir de la gestión compartida de recursos, de manera colaborativa e intergeneracional. Dentro de ADAN también hay docentes de la FIC que poseen una cuenta de usuario, algunos de ellos se registraron por invitación de los mismos estudiantes para participar y colaborar, y en ocasiones puntuales los mismos profesores utilizaron las salas virtuales para reunirse en el marco de sus investigaciones. ADAN tiene normas y reglas identificables dentro de su comunidad que le permite gestionar diversas herramientas tecnológicas9 "para que los estudiantes puedan compartir y producir conocimiento colectivo y prácticas pre-profesionales", sostienen sus administradores.

\section{Plataforma estudiantil: ADAN}

El 21 de septiembre de 2015 un profesor del Instituto de Comunicación (IC) de la FIC envió un mail a la lista docente informando:

Estimados colegas, reenvío un aviso que está circulando en Facebook entre nuestros estudiantes a los efectos de estar informados: 'Para la vida universitaria no alcanza solo con EVA, por eso surge ADAN, una plataforma colaborativa para que los estudiantes compartan propuestas de parciales y exámenes de la Facultad de Información y Comunicación.

Los comentarios de los docentes del IC en la cadena de mails dejaban al descubierto que el pacto comunicacional profesor-alumno, que proponía la plataforma EVA, estaba sufriendo alteraciones y generando una nueva configuración donde los estudiantes estaban participando de otros espacios virtuales no institucionales. Los alumnos parecían generar

9 TeamSpeak, Wordpress, gestores de proyectos, herramientas para diseño, edición y animación, capturadores de pantalla para tutoriales, google drive, programación, manejo de hosting a través de Cpanel, software para transmisiones en vivo de eventos o repasos, etc. Incluso la misma plataforma EVA. 
otras lógicas y estrategias colaborativas, participativas y de intercambio, a partir de la utilización de recursos tecnológicos, dentro del proceso educativo y por afuera de las herramientas propuestas en EVA para los mismos fines. Las lógicas participativas de los estudiantes del IC, organizados en la plataforma ADAN, interpelaba así a los docentes y a la misma UdelaR sobre los procesos de enseñanza tradicional dentro de la Universidad.

Los diversos dispositivos TIC, en mano de los estudiantes, parecían descentrar la relación del sistema educativo virtual y presencial oficial de EVA, subvirtiendo la premisa que se sostenía en el esquema clásico de enseñanza universitaria: el saber está centralizado y personificado en la figura del docente. Internet, a partir de la plataforma ADAN, permitía a los estudiantes, en principio, participar, cooperar e intercambiar, de forma horizontal entre sus miembros, en un espacio virtual y propio, poniendo en común dudas y consultas de exámenes. Investigar si las prácticas y usos de ADAN eran percibidas como transformadoras o "mejoristas" entre los docentes y estudiantes fue uno de los objetivos principales de este trabajo.

Es importante aclarar también que la idea de creación colectiva o aprendizaje colaborativo que desarrollan los estudiantes en la plataforma ADAN y/o EVA no es novedosa. El filósofo tunecino Pierre Lévy, uno de los referentes del campo de la cibercultura, explicaba en 1997 que el término Inteligencia colectiva refería a la capacidad de las comunidades virtuales de estimular el conocimiento y la pericia de sus miembros, a menudo mediante la colaboración y deliberación a gran escala. Lévy concibe la inteligencia colectiva como una nueva forma de poder. Un poder que cuestionaría la esencia misma del funcionamiento tradicional universitario y descentraría el poder del saber.

En la siguiente sección se presenta la metodología y luego los hallazgos más relevantes. Finalmente, en la conclusión, se sitúan las prácticas y las lógicas de la red como un espacio que resignifica e interpela las lógicas tradicionales universitarias, centralizadas y jerarquizada que ponen en tensión a los actores, docentes y estudiantes. 


\section{Metodología}

El objetivo general de la investigación es avanzar en el conocimiento sobre los sentidos y significados que adquieren para los estudiantes y los docentes el uso de las plataformas EVA y ADAN en el ámbito de las asignaturas de la FIC. Para ello se establecieron los siguientes objetivos secundarios:

- Identificar las potencialidades de uso de la plataforma EVA y ADAN.

- Describir los usos de las plataformas por parte de los estudiantes y docentes.

- Identificar las interacciones que se presentan en los usos de las plataformas EVA y ADAN.

- Analizar las normas y/o reglas por las que se rigen dichas interacciones.

Se optó por un estudio exploratorio, con un diseño metodológico de carácter cuantitativo y cualitativo, construido a partir de la combinación de cuatro procedimientos para la recopilación y constitución del corpus de análisis: a) se aplicó una encuesta on-line a estudiantes usuarios de la plataforma ADAN de la FIC, durante el período de 1 mes (de un universo de 1748 usuarios respondieron de forma voluntaria 19l-formularios válidos) cuyo análisis se realizó con la versión 19 de SPSS; b) entrevistas en profundidad10 a siete de los doce docentes de la FIC que participaron en la cadena de mails opinando sobre la creación de la plataforma estudiantil ADAN (el resto estaba fuera del país al momento de realizar las entrevistas), al secretario académico, un docente que no utiliza EVA y a dos expertos 11 de la plataforma EVA (realizadas en el período abril y mayo de 2016) que trabajan directamente con recursos abiertos en la UdelaR; c) observación del dictado de cuatro asignaturas

10 Las entrevistas pretenden indagar en los sentidos y significados que desarrollan los docentes en el uso de las plataformas EVA y Adán. Las categorías propuestas para el análisis son: 1) Espacio para el aprendizaje; 2) Participación; 3) Trabajo colaborativo; y 4) Percepción de Adán.

11 Las informantes calificadas fueron Virginia Rodés, Coordinadora del Programa de Entornos Virtuales de Aprendizaje de la Universidad de la República (ProEVA); y Patricia Díaz, integrante del Núcleo Interdisciplinario sobre Recursos Educativos Abiertos y abogada de Creative Commons Uruguay. 
en la plataforma EVAl2 para ahondar en las prácticas y dinámicas que los docentes realizan en los espacios virtuales de clase; d) análisis de datos secundarios de la plataforma ADAN (Google Analytic: dispositivos utilizados; interacciones con el sitio web; duración media de la visita; los flujos de la visita, etc.).

Las entrevistas permitieron reconstruir el discurso de docentes y expertos de la gestión oficial de la plataforma EVA que constituyen y construyen una argumentación que involucra la actividad de nombrar y clasificar, además de vincular contextos sociales y culturales, y que pueden excluir prácticas o formas de pensar alternativas.

Finalmente, las dimensiones de análisis sobre las que se trabajó fueron: a) inteligencia colectiva, como la capacidad de las comunidades virtuales de estimular el conocimiento y la pericia de sus miembros, a menudo mediante la colaboración y deliberación a gran escala; como una nueva forma de poder (LÉVY, 1997); b) participación, entendida como la negociación de las interacciones colaborativas en las cuales el individuo no impone su visión, participa argumentando su punto de vista y justifica, negocia e intenta convencer a sus pares; 3) bienes comunes 13, compuestos por tres elementos: los recursos, las comunidades que comparten dichos recursos y las normas desarrolladas por dichas comunidades con el objeto de hacer sostenible todo el proceso.

\section{Resultados}

En el siguiente apartado se da cuenta de los resultados en relación a los sentidos y significados de las prácticas desarrolladas en las plataformas ADAN y EVA. Para ello se construyeron dos categorías de análisis: 1) Espacio para el aprendizaje y la Participación; y 2) Dinámica de intercambio: EVA-ADAN. En este apartado se da cuenta de los principales

10 En el caso de EVA se seleccionó la observación de cuatro asignaturas en base al criterio de los estudiantes, quienes nos comentaron sobre los docentes que más actividades realizaban dentro de esa plataforma.

11 Son características de los bienes comunes: a) los sistemas de gobernanza o de gestión compartida de recursos, b) los sistemas desarrollados por determinadas comunidades y c) los sistemas que tienen normas y reglas identificables. 
hallazgos en cada categoría a partir de los cuatro procedimientos metodológicos propuestos.

\section{Espacio para el aprendizaje y la Participación}

\section{Rol de las TIC en la enseñanza y el aprendizaje}

La mayoría de los docentes entrevistados consideran a las TIC como una herramienta cada vez más relevante dentro del ámbito de la educación, pero señalan que deben ocupar un lugar complementario dentro del sistema educativo, como un apoyo didáctico y no como el centro de atención.

En este sentido, la integrante del Departamento de Apoyo Técnico Académico, Comisión Sectorial de Enseñanza, Virginia Rodés, subraya en la entrevista que el papel de las tecnologías -como un accesorio para acompañar, ampliar, propiciar, favorecer las enseñanzas y aprendizajesno arregla la enseñanza ni mejora el aprendizaje: "la incorporación de la tecnología es un instrumento para, pero lo que estructura y define es el uso que se les da". Para ella significa un "espacio de integración, de participación voluntaria (...) una plataforma educativa (...) que comparte identidad institucional” (RODÉS et al., 2009, p. 6).

Mientras que algunos docentes manifiestan en las entrevistas que la implementación de la plataforma EVA fue casi impuesta, verticalmente, por la UdelaR y la FIC. Para otros pocos, las plataformas responden más a una moda que a un apoyo didáctico y se corre el riesgo de subutilizar la herramienta.

La plataforma EVA, por más que se propuso como un espacio de integración, aún no logró que los estudiantes ni los profesores se apropiaran de la misma, y por tanto no hubo un cambio en las prácticas culturales ni de participación estudiantil.

En cambio, la implementación de ADAN por parte de los estudiantes, en el espacio virtual de las plataformas educativas, interpela a la Universidad a pensar en la reconfiguración de las prácticas culturales de los sujetos que integran las comunidades académicas, particularmente, 
sus estudiantes. La apropiación de los estudiantes de ADAN comporta un proceso que muestra las formas por medio de las cuales los estudiantes hacen suya la tecnología y la incorporan creativamente al conjunto de sus actividades cotidianas.

\section{Prácticas educativas con TIC: EVA-ADAN}

La plataforma EVA, según se desprende de las entrevistas, se utiliza esencialmente como repositorio de materiales. Algunos docentes consideran que EVA ha sido fundamental para democratizar el acceso a ciertos materiales de estudio. Tales percepciones se confirman en los datos del censo realizado a estudiantes de grado y posgrado en 2012 por los mismos coordinadores de Pro-EVA, donde la descarga de materiales es mencionada en el 98\% de los casos. En palabras de Rodés “(...) Entonces lo que tenemos es que los docentes lo que hacen es crear repositorios y los estudiantes lo que hacen es descargar material".

En otras palabras, una herramienta Moodle tan potente como EVA estaría siendo sub-utilizada, tal como se desprende de las asignaturas observadas para este estudio y de las entrevistas a los docentes, así como de la percepción de los propios administradores de la plataforma EVA. También existe entre los docentes la percepción de que EVA funciona como un canal de información y que facilita el intercambio de novedades, preguntas y notificaciones. Los estudiantes confirman que utilizan EVA como repositorio de materiales, y a la plataforma ADAN como un lugar complementario para acceder a clases grabadas y pautas de examen, por ejemplo. ADAN también funciona como repositorio de materiales, pero generados por los propios estudiantes y muchos de ellos trabajados de forma colectiva en ese espacio virtual. Según la encuesta, un $75,4 \%$ perciben a ADAN como un espacio de intercambio con otros estudiantes donde se puede aprender y aportar. Las prácticas de los estudiantes en ADAN estarían mostrando un nivel de apropiación y participación, pertenencia y compromiso que no logró la plataforma EVA, a saber: dominio de un objeto cultural que involucra el reconocimiento de la actividad que condensa la plataforma y con ella los sistemas de 
motivaciones, el sentido cultural del conjunto y prácticas específicas que implica su uso culturalmente organizado.

\section{Plataforma virtual y roles docente/estudiante}

La mayoría de los docentes entrevistados son categóricos en este punto y señalan que la relación profesor-estudiante no se reconfiguró ni transformó por las incidencias de las plataformas virtuales, como tampoco la participación de los estudiantes en los espacios virtuales o en el aula. Para ellos, el estudiante activo en el aula también lo es en EVA. Afirman que no depende de la creación de nuevos espacios, sino de las actitudes de los estudiantes. En otras palabras, algunos docentes no estarían de acuerdo con la idea de que un estudiante pasivo en clase luego sea activo en las aulas virtuales de la plataforma EVA. Sin embargo, otros profesores sostienen que el comportamiento activo o pasivo del estudiante depende de las características de cada curso, por ejemplo: si son clases masivas o no, si corresponden al ciclo de graduación u otro, etc. Los estudiantes encuestados perciben, en su mayoría, que su participación es en general "pasiva” (62,3\%), mientras que un 30\% de estudiantes se consideran "activos".

El docente sigue manteniendo una relación vertical con el alumno en ambos espacios de aprendizaje, en el aula y en EVA. Para Rodés, coordinadora de EVA, "no hay y no sé si debería haber un desdibujamiento de los roles docente-estudiante, creo que es una percepción que tuvimos en algún momento de desconocer la asimetría intrínseca que existe entre los roles docente-estudiante, una visión un poco idealista de la pedagogía”.

Sin embargo, Salinas (2004) entiende que el profesor debe dejar de ser fuente de todo conocimiento y pasar a actuar como guía de los estudiantes, acentuar su papel de orientador y mediador, facilitarles el uso de los recursos y las herramientas que necesitan para explorar y elaborar nuevos conocimientos y destrezas. Las nuevas formas de alfabetización digital, sostiene Alcoceba Hernando, parecen haber generado un desajuste y confusión dentro del profesorado sobre el uso de las TIC y las transformaciones en los modelos de enseñanza-aprendizaje: 
El modelo educativo basado en soportes expresivos escritos (libros) y en el principio de autoridad del profesorado (como acumulador y difusor de conocimiento) se ha transformado radicalmente en otro en que la información fluye de forma accesible a través de múltiples cauces y soportes: donde el alumnado no se limita a memorizar y acumular conocimiento, sino que debe seleccionar y ordenar la información de forma crítica; y donde el profesorado ya no aparece como un mero depositario del conocimiento, sino que se debe convertir en un gestor u orientador de los procesos de enseñanza a partir de los recursos tecnológicos (ALCOCEBA HERNANDO, 2013, p. 195).

La plataforma ADAN, integrada en su génesis mayoritariamente por estudiantes, constata transformaciones en los modelos de enseñanza, aún en estado germinal, donde se observa un nivel de participación diferencial al de la plataforma EVA. Son los estudiantes quienes se preocupan de que los materiales estén a disposición de todos, discuten conjuntamente respuestas y textos, responden de forma inmediata dudas de otros estudiantes que ingresan por primera vez. No existe en la plataforma una relación formal de profesor-estudiantes, sino una relación solidaria entre iguales en la cual los bienes no son individuales (apuntes, grabaciones, textos, etc.). La propiedad compartida de los bienes comunes hizo que los estudiantes desarrollaran formas innovadoras de organizar y trabajar los materiales de todo el colectivo creando al mismo tiempo un espacio basado en la amistad, intereses, prácticas y zonas compartidas, cuyos intercambios están sujetos a un estatuto común. Los estudiantes encuestados perciben que en ADAN se realizan actividades de estudio colaborativo (60\%) y que son escuchadas sus dudas y sugerencias (34\%). Se desprende de estos resultados que la plataforma ADAN estaría generando un sentido de pertenencia dentro del colectivo, a partir de nuevas formas de relacionamiento, donde el encuentro con amigos y colegas se re-configura y pasa a ser esencial para ser un sujeto integrado a un círculo o grupo de referencia. 


\section{Interacción y gestión de las plataformas}

Los profesores perciben una escasa interacción entre los propios estudiantes en la plataforma EVA y señalan que la interacción entre docentes y estudiantes es difícil debido a la gestión del tiempo, el esfuerzo y la dedicación que se requiere para aprovechar ese espacio virtual. En el caso de un curso semipresencial, por ejemplo, el docente no solo debe realizar un mayor seguimiento, sino también estimular a los estudiantes para que participen en la plataforma y dar cuenta de una rápida respuesta a las demandas de los alumnos.

Cuando se le pregunta a los docentes si tienen interés en obtener más provecho de EVA señalan que el tiempo y la dedicación que exige no están a su alcance. Algunos docentes también apuntan que los cursos del primer ciclo, donde la masividad de las clases es señalada como un gran problema, la gestión del tiempo para dar respuesta a todos los estudiantes es prácticamente inviable. Apuntan que, finalmente, los grados docentes más altos descargan las tareas en los grados más bajos, quedando en éstos últimos con la responsabilidad de gestionar y administrar las tareas e interacciones en la plataforma EVA.

En el caso de ADAN la gestión no recae sobre un estudiante sino sobre una comisión administradora de alumnos de la plataforma, quienes pueden hacer un rápido seguimiento a las demandas de sus pares porque gestionan tiempo y "bienes comunes" (SUBIRATS, 2012, 2013; BARBIERI, 2014; HOLDER; FLESSAS, 2008; OSTROM, 1990; BENKLER, 2006) en beneficio de la comunidad. Barbieri (2014, p. 111) esquematiza la idea de los bienes comunes como:

En primer lugar, debemos entender que los bienes comunes no son ni espacios ni objetos. Los bienes comunes están compuestos por tres elementos: unos recursos, unas comunidades que comparten dichos recursos y unas normas desarrolladas por dichas comunidades con el objeto de hacer sostenible todo el proceso. Es decir, los bienes comunes son: a) sistemas de gobernanza o de gestión compartida de recursos, b) sistemas desarrollados por determinadas comunidades, y c) sistemas que tienen normas, reglas identificables. Esto quiere decir que hemos aprendido a 
mirar los bienes comunes menos como sustantivos (el bien común) y más como adjetivos (lo común).

Se desprende de los datos que la plataforma EVA tendría un modelo de políticas de acceso (democratizar la información), con docentes y técnicos de la universidad que ejercerían un rol administrativo, vertical, de mando y control. Por lo tanto, la tarea que asume EVA sería la de promover una interacción de tipo racional instrumental. En cambio, el modelo de política de los bienes comunes es más próximo a la plataforma ADAN, donde los estudiantes se reconocen como interdependientes, horizontales, cooperativos, comunitarios y desarrollan, al mismo tiempo, instrumentos para promover la colaboración y la implicación.

Las nuevas formas de socialización de los estudiantes necesitan espacios más flexibles y dinámicos. La transformación en las formas de relación entre pares, horizontal, a partir de la irrupción de las TIC, supuso un cambio importante en el ámbito de la sociabilización de los jóvenes estudiantes. Las relaciones mediadas tecnológicamente en entornos virtuales como ADAN parece que reconfiguró nuevas formas de interacción que entran en tensión con las formas tradicionales, como las que se replican en la plataforma EVA, y que generan lazos de pertenencia y corresponsabilidad colaborativa.

Las formas tradicionales de aprendizaje dentro del sistema educativo universitario deben pensarse desde un enfoque diferente para entender los nuevos modos de relación entre los estudiantes y la apropiación del conocimiento. Una perspectiva que permita escapar a los esquemas clásicos de enseñanza y dé cuenta de las nuevas prácticas y estrategias de inteligencia colectiva -en la idea de Lévy- por parte de los estudiantes a partir de la flexibilidad de la red, de su capital cultural y simbólico en relación con la figura del docente como dinamizador y organizador del conocimiento. 


\section{Dinámica de intercambio: EVA-ADAN}

La mayoría de los docentes están al corriente de la existencia de ADAN, pero no todos conocen exactamente su funcionamiento. Algunos profesores afirman haber ingresado en la plataforma (deben registrarse con un usuario y una contraseña), pero dicen no haber navegado en ella. Los expertos de EVA expresan una visión positiva de la plataforma ADAN, y apuntan que la misma surgió en respuesta a necesidades estudiantiles que no fueron resueltas institucionalmente.

Para algunos profesores ADAN no es diferente a las prácticas de intercambio que los estudiantes realizaban antes de Internet. Sin embargo; opinan que el potencial de las prácticas en la plataforma desafía el trabajo docente. Se refieren al intercambio de las pautas de evaluación elaboradas por los profesores para los parciales/exámenes y que los estudiantes "cuelgan" en ADAN para discutirlas de forma colectiva, así como los audios de las clases grabadas en formato digital. La grabación de las clases abrió un debate dentro de la comunidad docente sobre: a) la privacidad, la ética de registrar sin consentimiento las clases, b) los derechos de autor y c) la propiedad intelectual de lo que se registra en las clases, entre muchos otros. Para algunos profesores existe una violación a la confidencialidad mientras que para otros el problema es difundir el audio "fuera de su contexto". Los pactos comunicacionales, explican los profesores, no son los mismos dentro del aula que fuera de ella, por lo que el sentido puede quedar sesgado.

Los docentes, en principio, estarían de acuerdo con las prácticas realizadas en ADAN, pero cuando se profundiza en las entrevistas sobre temas puntuales, como las clases grabadas sin consentimiento o la publicación de las pautas de examen, surge la discusión sobre la libertad de expresión e información y la regulación de ciertas prácticas en la plataforma estudiantil. Para algunos profesores la regulación puede atentar contra tales libertades. En cambio, otros docentes entienden que se necesitaría algún tipo de reglamentación que garantice, por ejemplo, el derecho de autor o la libertad de cátedra. A pesar de ello creen difícil e inviable la normativización y preferirían abrir un debate conjunto entre 
estudiantes y docentes para discutir las incidencias de ciertas prácticas y el alcance ético de las mismas.

Es en este punto, a partir de las subjetividades de los docentes, donde se visualiza uno de los conflictos y tensiones más importantes en la gestión de ambas plataformas. Por un lado, uno de los objetivos principales de EVA es generar un modelo de políticas de acceso (democratizar la información) y cuando los estudiantes de ADAN llevan adelante tal política de acceso, para los docentes es percibido como un problema. Sin embargo, los docentes no tienen inconvenientes en subir a EVA textos, películas, capítulos de libro, libros enteros, etc. Tal práctica no es autocuestionada por los docentes ni entra en contradicción con los dilemas éticos y/o legales como la propiedad intelectual o los derechos de autor que mencionaban respecto a la plataforma ADAN.

\section{Conclusión}

Este trabajo pretende abrir un diálogo con otras investigaciones que aborden las TIC en relación a los procesos de apropiación, circulación y producción de conocimiento, poniendo en tensión las formas tradicionales de pensar la enseñanza y otras formas de participación estudiantil que interpelan las mediaciones y las lógicas centralizadas, verticales y jerarquizadas del sistema universitario.

El estudio muestra que un porcentaje importante de los estudiantes estarían aprovechando sus conocimientos individuales en pro de metas y objetivos compartidos en el sentido que advertía Lévy, nadie lo sabe todo, todo el mundo sabe algo. Los estudiantes organizados en la plataforma ADAN trabajan de forma colectiva para acceder a un saber al que no podrían llegar por ellos mismos. Esa organización les deja generar una comunidad de conocimientos, término también usado por Lévy, que les permite discutir, negociar y lograr un desarrollo colectivo y colaborativo, además de estimularlos de forma individual para buscar nueva información en pos del bien común.

Es importante señalar que los estudiantes que conforman ADAN en ningún momento cuestionan la figura del docente y siguen buscando 
en ella la orientación y organización del conocimiento. A cambio les demandan a los profesores la legitimación de sus prácticas y los invitan a participar de un espacio en el que ellos, de forma autónoma, gestionan y administran unos bienes comunes que comparten de forma colectiva. No se desprende de los datos de las encuestas que los estudiantes busquen en sus prácticas en red reemplazar al docente ni el espacio tradicional de la clase universitaria. Al contrario, los estudiantes que integran ADAN pidieron a los docentes que participaran en sus debates, que corrigieran los apuntes que compartían de forma colaborativa de sus asignaturas y que organizaran el material de sus clases. Además, en sus prácticas de estudios acaban generando una lista de dudas que se trasladan al profesor en los espacios de consulta generados a tales efectos. Los sentidos y significados de las prácticas de lecturas colaborativas en ADAN, en última instancia, terminan siendo validadas por el docente en el aula o en dichos espacios.

El desafío de la gestión del sistema universitario es entender los modos de relación, apropiación, los procesos de referencialidad y la creciente complejidad comunicacional que se tejen en esas plataformas virtuales entendidas como nuevos espacios tecnológicos, geográficos y sociales. Las posibilidades abiertas por las TIC en la construcción colectiva, colaborativa y horizontal del desarrollo de la inteligencia conectiva (a partir de la interacción estudiantil con los sistemas modernos de comunicación, con características autonómicas y con un modelo de producción y gestión sostenido en el bien común), nos obliga a reflexionar sobre el modelo tradicional educativo. Modelo, este último, basado en el esquema clásico de enseñanza universitaria y en el cual el saber conserva un carácter jerárquico, centralizado en el espacio-tiempo y personificado en figuras concretas.

El sistema universitario no puede quedar ajeno a estos cambios que permiten hoy, como nunca antes, que los estudiantes tengan cada vez más control de la producción del campo simbólico, de su propia narrativa y modelo de representación, ya que pasaron de consumidores a creadores culturales. Los estudiantes son protagonistas en la era de 
Internet, se apropiaron de las transformaciones comunicacionales que trajeron las TIC en la conformación de un nuevo orden mediático, renunciaron a ser sólo espectadores para hablar, decir y representar. La universidad deberá innovar en sus modelos de enseñanza y participación para favorecer la calidad de la intervención estudiantil en el marco de un nuevo círculo virtuoso de enseñanza y desarrollo.

\section{Referencias bibliográficas}

ALCOCEBA HERNANDO, J. Juventud, tecnologías de la información y cambio social. Perspectivas y escenarios para la socialización y la participación. In: CABALLERO, F. (Org.). Ciudadanía, tecnología y cultura: nodos conceptuales para pensar la nueva mediación digital. Barcelona: Gedisa, 2013. p. 181-210.

BARBIERI, N. Cultura, políticas públicas y bienes comunes: hacia unas políticas de lo cultural. Kult-ur, n. 1, p. 101-119, 2014. Disponível em: <http://www.e-revistes.uji.es/ index.php/kult-ur/article/view/1255/1232>. Acesso em: out. 2016.

BENKLER, Y. The Wealth of Networks: how social production transforms markets and freedom. New Haven: Yale U. Press, 2006.

CASTELLS, M. La era de la información. Madrid: Alianza, 1997.

DARLING-HAMMOND, L. El derecho de aprender: crear buenas escuelas para todos. Barcelona: Ariel, 2001

CROVI DRUETTA, D. Retos de las universidades en la sociedad de la información y el conocimiento. In: CABELLO, R.; LEVIS, R. (Orgs.). Medios informáticos en la educación a comienzos del siglo XXI. Buenos Aires: Prometeo, 2007.

DE MORAES, D. Comunicação alternativa, redes virtuais e ativismo: avanços e dilemas. Eptic, v. IX, n. 2, mai./ago. 2007. Disponível em: <http://observatoriodaimprensa.com. br/wp-content/uploads/2015/02/Comunicacao_alternativa-1.pdf>. Acesso em: out. 2016. ECHEVERRIA, J. Los señores del aire: Telépolis y el Tercer Entorno. Barcelona: Destino, 1999.

FISCHER, H. Le choc numerique. A l'aube d'une nouvelle civilisation. Montreal: VlbEditor, 2001.

HOLDER, J.; FLESSAS, T. Emerging Commons. Social \& Legal Studies, n. 17. p. 299-311, 2008.

LÉVY, P. Collective intelligence: mankind's emerging world in cyberspace. Cambridge: Perseus Books, 1997. 
MARTÍN BARBERO, J. Tecnicidades. Identidades, alteridades, des-ubicaciones y opacidades de la comunicación en el nuevo siglo. Diálogos de la Comunicación, Guadalajara, n. 64, p. 8-23. 2007. Disponível em: <http://www.olavarria.com/ciudad/universitarios/ biblioteca/descargas/b/martin_barberol.pdf>. Acceso em: out. 2016.

MEC. Dirección de Educación. Panorama de la educación 2014. Montevideo: MEC. Dirección de Educación, 2014. Disponível em: <http://www.mec.gub.uy/innovaportal/ file/11078/1/mec-panorama-educacion-2014.pdf > Acceso en: mai. 2017.

OSTROM, E. Governing the Commons: The Evolution of Institutions for Collective Action. London: Cambridge University Press, 1990.

RODÉS, V.; PINTOS, G.; PÉREZ, A.; CORREA, N.; BUDIÑO, G.; PERÉ, N.; ALONZO, L.; DOS SANTOS, S. Análisis de procesos de cambio tecnológico y organizacional para la integración de TIC en la Universidad de la República. In: XIII CONGRESO INTERNACIONAL DE EDUCACIÓN A DISTANCIA. CREAD - UDEC /MERCOSUR/SUL. Anais... Concepción, Chile, 2009. Disponível em: <http://reposital.cuaed. unam.mx:8080/jspui/bitstream/123456789/2898/1/Rod\%C3\%A9s,\%20Virginia.pdf>.

Acesso em: out. 2016

RIFKIN, J. La era del acceso. Barcelona: Paidós, 2000.

SALINAS, J. Innovaciones docentes y uso de las TIC en la enseñanza universitaria. Revista de Universidady Sociedad del Conocimiento (RUSC), v. 1, 2004. Disponível em: $<$ https://www.uoc.edu/rusc/dt/esp/salinas1 104.pdf>. Acesso em: abr. 2017.

SUBIRATS, J. Nuevos tiempos. ¿Nuevas políticas públicas? Explorando caminos de respuesta. In: XVII Congreso Internacional del CLAD sobre la Reforma del Estado y de la Administración Pública, Cartagena. Anais... Colombia, 2012. Disponível em: <http:// www.dgsc.go.cr/dgsc/documentos/cladxvii/subirats-conf.pdf>. Acesso em: out. 2016.

SUBIRATS, J. Bienes comunes y contemporaneidad. Releyendo a Polanyi. Ecología Política, cuadernos de debate internacional. 2013. Disponível em: <http://ecologiapolitica. info/wordpress/?p=913>. Acesso em: out. 2016.

\section{Sobre os autores}

Mauricio Nihil Olivera Cajiga - Profesor Adjunto e Investigador (SNI-ANII) Facultad de Información y Comunicación Departamento de Medios y Lenguajes Sección académica Sociedad, Cultura y TIC Universidad de la República.

María Julia Morales - Coordinadora del ObservaTIC, Facultad de Ciencias Sociales-UdelaR. 
112 PLATAFORMAS VIRTUALES: ¿HERRAMIENTAS PARA EL APRENDIZAJE?

Analía Passarini - Profesora Facultad de Información y Comunicación Departamento de Medios y Lenguajes Sección académica Sociedad, Cultura y TIC Universidad de la República.

Natalia Correa - Profesora Facultad de Información y Comunicación Departamento de Medios y Lenguajes Sección académica Sociedad, Cultura y TIC Universidad de la República.

Fecha de envío: 09/01/2017

Fecha de aceptación: 24/04/2017 